\title{
Electric Field Distributions in the Open Cylindrical Silicon Carbide Waveguides
}

\author{
S. Asmontas, L. Nickelson* And T. Gric \\ Semiconductor Physics Institute, A. Goštauto 11, Vilnius, Lithuania
}

\begin{abstract}
Here are presented the results of an electrodynamical analysis of the $\mathrm{SiC}$ waveguides with two different radii $R=1.5 \mathrm{~mm}$ and $R=2.5 \mathrm{~mm}$. We have investigated the dispersion characteristics of these waveguides as well as the electric field distributions in the waveguide cross-sections at $f=50$ and $25 \mathrm{GHz}$.
\end{abstract}

PACS numbers: 75.47.Lx, 75.47.Gk, 73.50.Fq

\section{Introduction}

Progress in the build-up of high power microwave devices stimulates the intensive research of the open silicon carbide ( $\mathrm{SiC}$ ) semiconductor waveguides. SiC waveguides operating at the microwave range are presently being developed for advantageous use in high-temperature, high-power, and high-radiation conditions. We are going to present here results of electrodynamical analysis of the open cylindrical $\mathrm{SiC}$ waveguide. We have created a computer algorithm with $2 \mathrm{D}$ graphical visualization in the MATLAB language.

\section{Numerical results}

We have analyzed electrodynamical characteristics of the open circular cylindrical $\mathrm{SiC}$ semiconductor waveguide (Fig. 1). The solution of this electrodynamical problem and the approbation of our computer algorithm are given in [1]. The numerical results of the $\mathrm{SiC}$ waveguide investigation are presented at the temperatures $T=20^{\circ} \mathrm{C}$ and $T=1800^{\circ} \mathrm{C}$. The permittivities of $\mathrm{SiC}$ material are $\varepsilon_{\mathrm{r}}^{\mathrm{SiC}}=6-0.5 \mathrm{i}$ at $T=20^{\circ} \mathrm{C}$ and $\varepsilon_{\mathrm{r}}^{\mathrm{SiC}}=11-7 \mathrm{i}$ at $T=1800^{\circ} \mathrm{C}[2]$. In the present article the azimuthal index characterizing azimuthal variations of the electric field is $m=1$.

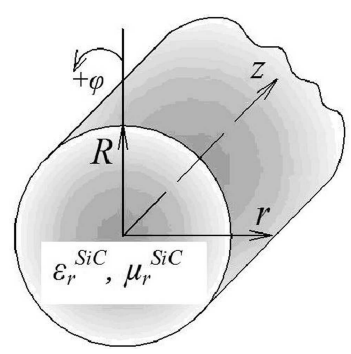

Fig. 1. The $\mathrm{SiC}$ waveguide model.

\footnotetext{
* corresponding author; e-mail: lucynickelson@gmail.com
}

In Fig. 2 we can see the dispersion characteristics of the $\mathrm{SiC}$ waveguides with radii $R=1.5 \mathrm{~mm}$ (a) and $R=2.5 \mathrm{~mm}$ (b) at $T=20^{\circ} \mathrm{C}$. Here, the magnitude $h$ is the longitudinal propagation constant, $k$ is the wave number in free space, $f$ is an operating frequency. In Fig. 2 the designation 0 means the main mode, designations 1, 2, 3 mean the first, second and third higher modes, correspondingly.

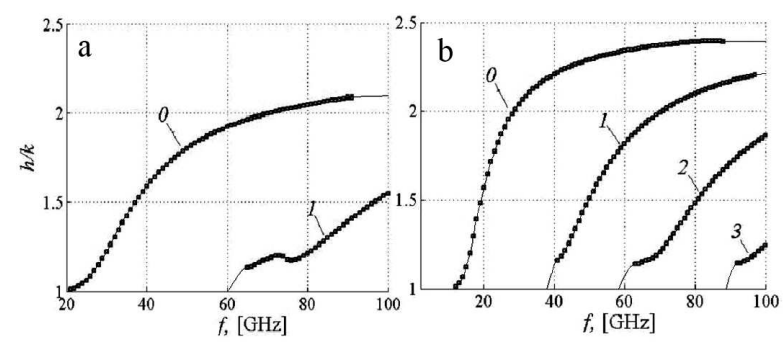

Fig. 2. Dispersion characteristics of the $\mathrm{SiC}$ waveguides when radii are $R=1.5 \mathrm{~mm}$ (a) and $R=$ $2.5 \mathrm{~mm}(\mathrm{~b})$.

We see that two hybrid slow modes can propagate in the $\mathrm{SiC}$ waveguide with the smaller radius $(R=1.5 \mathrm{~mm})$ and the number of the propagating modes increases twice when the radius of the $\mathrm{SiC}$ waveguide is larger $(R=2.5 \mathrm{~mm})$. We see that the $\mathrm{SiC}$ waveguide with the smaller radius has a larger bandwidth in comparison to the waveguide with the larger radius.

The electric field distributions of the main mode of the $\mathrm{SiC}$ waveguide at the operating frequency $f=50 \mathrm{GHz}$ are presented in Figs. 3, 4. The electric field calculations were executed in 10000 points of the waveguide cross-section. The electric field strength lines are presented in Figs. 3a-5a. Visualizations of the electric field intensity are shown in Figs. 3b-5b. The electric field distributions of the main hybrid mode of the $\mathrm{SiC}$ waveguide with $R=1.5 \mathrm{~mm}$ are shown in Fig. 3 .

In Fig. 3 we can see that the electric field concentrates in two places. The strongest electric field is in the small 


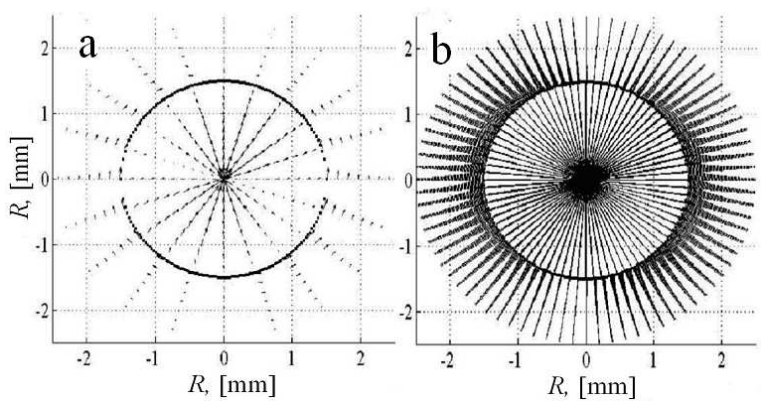

Fig. 3. The electric field distributions of the main mode propagating in the $\mathrm{SiC}$ waveguide with $R=1.5 \mathrm{~mm}$ at $T=20^{\circ} \mathrm{C}$. (a) the electric field strength lines, (b) the electric field intensity.
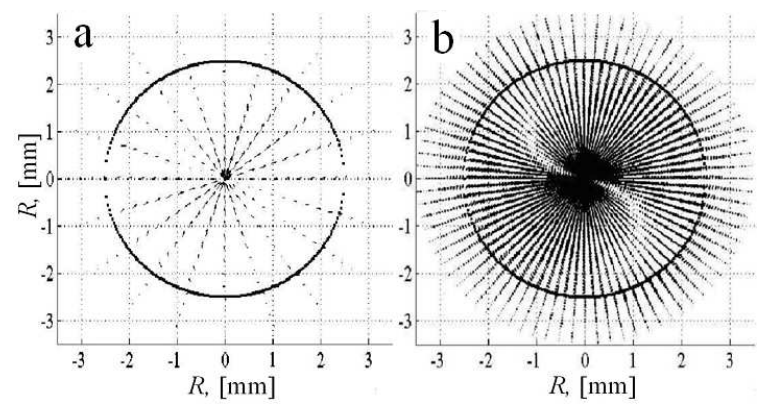

Fig. 4. The electric field distributions of the main mode propagating in the $\mathrm{SiC}$ waveguide with $R=2.5 \mathrm{~mm}$ at $T=20^{\circ} \mathrm{C}$. (a) the electric field strength lines, (b) the electric field intensity.

center area of the waveguide. We can see that the electric field is also stronger along the waveguide boundary.

The electric field distributions of the main hybrid mode of the $\mathrm{SiC}$ waveguide with $R=2.5 \mathrm{~mm}$ are presented in Fig. 4. We can see that the electric field concentrates only in the waveguide center. The electric field intensity has a shape of two turned lobes. We see that the electric field of the waveguide with $R=2.5 \mathrm{~mm}$ decreases quicker outside the $\mathrm{SiC}$ waveguide than the electric field outside the waveguide with $R=1.5 \mathrm{~mm}$. The comparison of the electric field distributions (Figs. 3 and 4) has pointed out that there is a difference between these two waveguides at the same operating frequency. This difference can be caused by different wavelengths $\lambda_{\mathrm{w}}=2 \pi / h$ of the main modes propagating in these waveguides. We can see that the main mode of the waveguide with $R=1.5 \mathrm{~mm}$ is described by $h / k=1.8$ and this mode of the waveguide with $R=2.5 \mathrm{~mm}$ is characterized by $h / k=2.3$ at $f=50 \mathrm{GHz}$ (Fig. 2). We see that the wavelength $\lambda_{\mathrm{w}}$ of the main mode propagating in the waveguide with the large radius is smaller in comparison to the wavelength $\lambda_{\mathrm{w}}$ of the main mode propagating in the waveguide with the smaller radius at the same frequency $f=50 \mathrm{GHz}$.

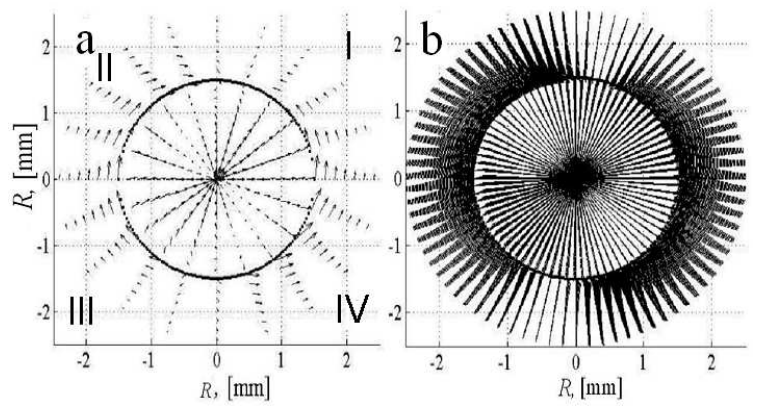

Fig. 5. The electric field distributions of the main mode propagating in the $\mathrm{SiC}$ waveguide with $R=1.5 \mathrm{~mm}$ at $T=1800^{\circ} \mathrm{C}$. (a) the electric field strength lines, (b) the electric field intensity.

In Fig. 5 we can see the electric field distribution of the main mode propagating in the $\mathrm{SiC}$ waveguide with radius $R=1.5 \mathrm{~mm}$ at $f=25 \mathrm{GHz}$.

In Fig. 5a we can see that the electric field strength lines outside the waveguide are directed clockwise in the II and III quarters and counter-clockwise in the I and IV quarters. We should notice that the electric field distribution of the main slow mode depicted in Fig. 5 is rotated by 90 degrees respectively to the electric field distribution of the main mode propagating in an analogical waveguide made of lossless material with the permittivity equal to $11[3]$.

\section{Conclusions}

We have presented here the electrodynamical characteristics of two cylindrical $\mathrm{SiC}$ waveguides with radii $1.5 \mathrm{~mm}$ and $2.5 \mathrm{~mm}$. We have found out the difference between the electric field distributions of the hybrid main modes propagating in these waveguides at $f=50 \mathrm{GHz}$. The electric field of the main mode propagating in the waveguide with $R=2.5 \mathrm{~mm}$ concentrates only in its center and quickly decreases outside this waveguide. Therefore the breakdown energy can be higher in the waveguide with $R=2.5 \mathrm{~mm}$ in comparison to the waveguide with $R=1.5 \mathrm{~mm}$. The electric field is stronger outside the $\mathrm{SiC}$ waveguide at $T=1800^{\circ} \mathrm{C}$ (the material permittivity is $\left.\varepsilon_{\mathrm{r}}^{\mathrm{SiC}}=11-7 \mathrm{i}\right)$ than the electric field outside the $\mathrm{SiC}$ waveguide at $T=200^{\circ} \mathrm{C}\left(\varepsilon_{\mathrm{r}}^{\mathrm{SiC}}=6-0.5 \mathrm{i}\right)$.

\section{References}

[1] L. Nickelson, T. Gric, S. Asmontas, R. Martavicius, Electron. Electr. Eng. 2, 82 (2008).

[2] T.A. Baeraky, Egypt. J. Solids 25, 263 (2002).

[3] L. Nickelson, V. Shugurov, Singular Integral Equations' Methods for the Analysis of Microwave Structures, Leiden, Boston 2005, p. 348. 